\title{
Completeness of context-sensitive rewriting ${ }^{\text {th }}$
}

\author{
Salvador Lucas ${ }^{\mathrm{a}, \mathrm{b}}$ \\ ${ }^{a}$ CS Dept. at the University of Illinois at Urbana-Champaign \\ ${ }^{b}$ DSIC, Universitat Politècnica de València
}

\begin{abstract}
Restrictions of rewriting may turn normal forms of some terms unreachable, leading to incomplete computations. Context-sensitive rewriting (CSR) is the restriction of rewriting that only permits reductions on arguments selected by a replacement map $\mu$, which associates a subset $\mu(f)$ of argument indices to each function symbol $f$. Hendrix and Meseguer defined an algebraic semantics for Term Rewriting Systems (TRSs) executing CSR that can be used to reason about programs written in programming languages like CafeOBJ and Maude, where such replacement restrictions can be specified in programs. Semantic completeness of CSR was also defined. In this paper we show that canonical replacement maps, which play a prominent role in simulating rewriting computations with CSR, are necessary for completeness in important classes of TRSs.
\end{abstract}

Keywords: algebraic semantics, context-sensitive rewriting, formal reasoning

\section{Introduction}

Recursive definitions that use conditional expressions, often require the use of syntactic restrictions to obtain terminating programs.

Example 1. The following TRS encodes a definition of the factorial function:

$$
\begin{aligned}
& \mathrm{p}(\mathrm{s}(x)) \rightarrow x \quad \text { zero }(0) \rightarrow \text { true } \quad \text { zero }(\mathrm{s}(x)) \rightarrow \text { false } \\
& 0+x \rightarrow x \quad 0 \times y \rightarrow 0 \quad \text { if(true, } x, y) \rightarrow x \\
& \mathrm{~s}(x)+y \quad \rightarrow \quad \mathrm{s}(x+y) \quad s(x) \times y \quad \rightarrow \quad y+(x \times y) \quad \text { if }(\text { false, } x, y) \quad \rightarrow \quad y \\
& \operatorname{fact}(x) \rightarrow \operatorname{if}(\operatorname{zero}(x), \mathrm{s}(0), \operatorname{fact}(\mathrm{p}(x)) \times x)
\end{aligned}
$$

Without any restriction on the evaluation of the arguments of if, the last rule makes the program nonterminating. Most implementations first (or just) evaluate the boolean condition and restrict the evaluation of the other arguments.

In context-sensitive rewriting (CSR $[4,5])$, fixed restrictions on reductions are imposed by means of a replacement map $\mu$ that, for each $k$-ary symbol $f$, specifies

\footnotetext{
${ }^{\sqrt{2}}$ Developed during a sabbatical year at UIUC. Supported by NSF CNS 13-19109, MINECO project TIN2010-21062-C02-02, GV grant BEST/2014/026 and PROMETEO/2011/052.
}

Preprint submitted to Information Processing Letters

July 11, 2014 
the argument positions $i \in \mu(f) \subseteq\{1, \ldots, k\}$ which can be rewritten. We say that a replacement map is compatible with a given rule $\ell \rightarrow r$ of a TRS $\mathcal{R}$, if the positions of nonvariable symbols in $\ell$ are always reducible under $\mu$. We say that $\mu$ is a canonical replacement map if it is compatible with all rules of the TRS $\mathcal{R}$. The use of canonical replacement maps $\mu$ ensures that context-sensitive computations may stop yielding head-normal forms, values or even normal forms $[4,5]$. With $\mu$ (if $)=\{1\}$ we obtain a terminating behavior for $\mathcal{R}$ in Example 1 which can be proved with existing termination tools like MU-TERM. Indeed, CSR can compute the value $\mathrm{s}^{n !}(0)$ of any call fact $\left(\mathrm{s}^{n}(0)\right)$, for $n \geq 0$, without running in any termination problem (Example 4). In contrast, a normalizing evaluation strategy (e.g., the leftmost-outermost rewriting strategy, which is normalizing for $\mathcal{R}$ ) does not stop with terms like fact $(\mathrm{p}(0))$ having no normal form.

Recently, several authors have investigated semantic properties of computations with CSR $[3,6]$. The motivation is devising appropriate models to (inductively) reason about properties of programs of programming languages like CafeOBJ [2] or Maude [1], where the specification of context-sensitive replacement restrictions is allowed. Hendrix and Meseguer introduce a number of semantic properties ( $\mu$-canonical completeness, $\mu$-semantic completeness and $\mu$-sufficient completeness) that can be used to guarantee that CSR is well-suited to tackle the desired formal framework for reasoning about programs with replacement restrictions. The results in [3] are completely general and do not refer to any specific class of TRSs or replacement maps. This is in sharp contrast with the analysis of completeness of CSR in $[4,5]$, where left linearity of TRSs and canonicity of replacement maps are required. In this paper we show that, for orthogonal TRSs, the use of a canonical replacement map is necessary for enjoying the three previous semantic properties. For canonical completeness, it is also necessary that all arguments of all constructor symbols be $\mu$-replacing (not only with orthogonal TRSs but with any TRS). Furthermore, being completely defined (i.e., ground normal forms contain no defined symbols) is also $n e c e s s a r y$ for TRSs $\mathcal{R}$ that enjoy the considered semantic properties.

\section{Preliminaries}

Given a set $A$, a binary relation $\mathrm{R} \subseteq A \times A$ is terminating if there is no infinite sequence $a_{1}, a_{2}, \ldots, a_{n}, \ldots$ such that for all $i \geq 1, a_{i} \in A$ and $a_{i} \mathrm{R} a_{i+1}$. In this paper, $\mathcal{X}$ denotes a countable set of variables and $\mathcal{F}$ denotes a signature, i.e., a set of function symbols $\{f, g, \ldots\}$, each having a fixed arity given by a mapping ar $: \mathcal{F} \rightarrow \mathbb{N}$. The set of terms built from $\mathcal{F}$ and $\mathcal{X}$ is $\mathcal{T}(\mathcal{F}, \mathcal{X})$. A term without variables is called ground. The set of ground terms is $\mathcal{T}(\mathcal{F})$. A term is said to be linear if it has no multiple occurrences of a single variable. Terms are viewed as labelled trees in the usual way. Positions $p, q, \ldots$ are represented by chains of positive natural numbers used to address subterms of $t$. We denote the empty chain by $\Lambda$. Given positions $p, q$, we denote its concatenation as $p . q$. Positions are ordered by the standard prefix ordering $\leq$. Given a set of positions $P$, minimal $_{\leq}(P)$ is the set of minimal positions of $P$ (ordered by $\leq$ ). If $p$ is a position, and $Q$ is a set of positions, $p \cdot Q=\{p \cdot q \mid q \in Q\}$. The set of positions of 
a term $t$ is $\operatorname{Pos}(t)$. Positions of non-variable symbols in $t$ are denoted as $\mathcal{P}_{\text {os }}(t)$, and $\operatorname{Pos}_{\mathcal{X}}(t)$ are the positions of variables. The subterm of $t$ at position $p$ is denoted as $\left.t\right|_{p}$ and $t[s]_{p}$ is $t$ with $\left.t\right|_{p}$ replaced by $s$. The symbol labelling the root of $t$ is denoted as $\operatorname{root}(t)$. A rewrite rule is an ordered pair $(\ell, r)$, written $\ell \rightarrow r$ (or $\alpha: \ell \rightarrow r$ if labelled $\alpha$ for further reference), with $\ell, r \in \mathcal{T}(\mathcal{F}, \mathcal{X}), l \notin \mathcal{X}$ and $\mathcal{V} a r(r) \subseteq \mathcal{V}$ ar $(l)$. The left-hand side (lhs) of the rule is $\ell$ and $r$ is the right-hand side $(r h s)$. A TRS is a pair $\mathcal{R}=(\mathcal{F}, R)$ where $R$ is a set of rewrite rules. $L(\mathcal{R})$ denotes the set of $l h s$ 's of $\mathcal{R}$. An instance $\sigma(l)$ of a lhs $l$ of a rule is a redex. The set of redex positions in $t$ is $\mathcal{P o s}_{\mathcal{R}}(t)$. If $\mathcal{P}_{o s}(t)=\varnothing$, then $t$ is a normal form. Let $\mathrm{NF}_{\mathcal{R}}$ (resp. $\mathrm{GNF}_{\mathcal{R}}$ ) be the set of (ground) normal forms of $\mathcal{R}$. A TRS $\mathcal{R}$ is left-linear if for all $l \in L(\mathcal{R}), l$ is a linear term. Given $\mathcal{R}=(\mathcal{F}, R)$, we consider $\mathcal{F}$ as the disjoint union $\mathcal{F}=\mathcal{C} \uplus \mathcal{D}$ of symbols $c \in \mathcal{C}$, called constructors and symbols $f \in \mathcal{D}$, called defined functions, where $\mathcal{D}=\{\operatorname{root}(l) \mid l \rightarrow r \in R\}$ and $\mathcal{C}=\mathcal{F}-\mathcal{D}$. Then, $\mathcal{T}(\mathcal{C}, \mathcal{X})$ (resp. $\mathcal{T}(\mathcal{C})$ ) is the set of (ground) constructor terms. A defined symbol $f$ is completely defined if there is no $t \in \mathrm{GNF}_{\mathcal{R}}$ such that $\operatorname{root}(t)=f$. A TRS $\mathcal{R}=(\mathcal{C} \uplus \mathcal{D}, R)$ is a constructor system (CS) if for all $f\left(\ell_{1}, \ldots, \ell_{k}\right) \rightarrow r \in R, \ell_{i} \in \mathcal{T}(\mathcal{C}, \mathcal{X})$, for $1 \leq i \leq k$. A term $t \in \mathcal{T}(\mathcal{F}, \mathcal{X})$ rewrites (in one-step) to $s$ (at position $p$ ), written $t \stackrel{p}{\rightarrow}_{\mathcal{R}} s$ (or just $t \rightarrow s$ ), if $\left.t\right|_{p}=\sigma(\ell)$ and $s=t[\sigma(r)]_{p}$, for some rule $\ell \rightarrow r \in R, p \in \mathcal{P} o s(t)$ and substitution $\sigma$. We say that $s$ rewrites to $t$ if $s \rightarrow^{*} t$. A TRS is terminating if $\rightarrow$ is terminating. A term is said to be normalizing if it rewrites into a normal form. A TRS $\mathcal{R}$ is normalizing if every term is normalizing. A TRS is called completely defined or exhaustive if no ground normal form contains a defined symbol.

Context-sensitive rewriting. A mapping $\mu: \mathcal{F} \rightarrow \wp(\mathbb{N})$ is a replacement map $\left(\mathcal{F}\right.$-map) if for all $f \in \mathcal{F}, \mu(f) \subseteq\{1, \ldots, \operatorname{ar}(f)\}[4] . \quad M_{\mathcal{F}}$ is the set of $\mathcal{F}$ maps. For a TRS $\mathcal{R}=(\mathcal{F}, R)$, we use $M_{\mathcal{R}}$ instead of $M_{\mathcal{F}}$. We write $\mu \sqsubseteq$ $\mu^{\prime}$ if for all $f \in \mathcal{F}, \mu(f) \subseteq \mu^{\prime}(f)$ and say that $\mu$ is more restrictive than $\mu^{\prime}$. We write $\left(\mu \sqcup \mu^{\prime}\right)(f)=\mu(f) \cup \mu^{\prime}(f)$ for all $f \in \mathcal{F}$. The set of $\mu$-replacing positions of $t$ is: $\mathcal{P o s}^{\mu}(t)=\{\Lambda\}$, if $t \in \mathcal{X}$ and $\mathcal{P o s}^{\mu}(t)=\{\Lambda\} \cup \bigcup_{i \in \mu(\operatorname{root}(t))} i \cdot \mathcal{P o s}^{\mu}\left(\left.t\right|_{i}\right)$ if $t \notin \mathcal{X}$. The set of non- $\mu$-replacing positions is $\overline{\mathcal{P}_{o s}{ }^{\mu}}(t)=\mathcal{P}$ os $(t)-\mathcal{P}_{o s}{ }^{\mu}(t)$. The non- $\mu$-replacing positions of $t$ have a frontier set $\mathcal{F} r^{\mu}(t)=$ minimal $_{\leq}\left(\overline{\mathcal{P}_{\text {os }}{ }^{\mu}}(t)\right)$ with the active positions. The maximal replacing context $M R C^{\mu}(t)=t[\square]_{\mathcal{F} r^{\mu}(t)}$ of $t$ is the part of $t$ whose positions are $\mu$-replacing in $t$ [5]. The canonical replacement map $\mu_{\mathcal{R}}^{\text {can }}$ of $\mathcal{R}$ is the most restrictive replacement map ensuring that the nonvariable subterms of the left-hand sides of the rules of $\mathcal{R}$ are $\mu$ replacing $[4,5]$ : for each $f \in \mathcal{F}$ and $i \in\{1, \ldots, \operatorname{ar}(f)\}, i \in \mu_{\mathcal{R}}^{\text {can }}(f)$ iff $\exists \ell \in$ $L(\mathcal{R}), p \in \mathcal{P o s}_{\mathcal{F}}(l),\left(\operatorname{root}\left(\left.l\right|_{p}\right)=f \wedge p . i \in \mathcal{P}_{\text {os }}(l)\right)$. Given a TRS $\mathcal{R}, C M_{\mathcal{R}}=$ $\left\{\mu \in M_{\mathcal{R}} \mid \mu_{\mathcal{R}}^{\text {can }} \sqsubseteq \mu\right\}$ is the set of replacement maps that are equal to or less restrictive than the canonical replacement map. If $\mu \in C M_{\mathcal{R}}$, we say that $\mu$ is a canonical replacement map for $\mathcal{R}$. Given a TRS $\mathcal{R}=(\mathcal{F}, R), \mu \in M_{\mathcal{R}}$, and $s, t \in \mathcal{T}(\mathcal{F}, \mathcal{X}), s \mu$-rewrites to $t$, written $s \stackrel{p}{\hookrightarrow_{\mathcal{R}, \mu}} t$ (or $s \hookrightarrow_{\mathcal{R}, \mu} t, s \hookrightarrow_{\mu} t$, or even $s \hookrightarrow t$ ), if $s \stackrel{p}{\rightarrow} \mathcal{R} t$ and $p \in \mathcal{P}_{o s}{ }^{\mu}(s)$ [4]. If $\mu \in C M_{\mathcal{R}}$, we often say that $\hookrightarrow_{\mu}$ performs canonical context-sensitive rewriting steps [5]. A term $t$ without replacing redexes (i.e., $\mathcal{P}_{o s}^{\mu}(t)=\varnothing$ ) is called a $\mu$-normal form, and $\mathrm{NF}_{\mathcal{R}}^{\mu}$ (resp. 
$\mathrm{GNF}_{\mathcal{R}}^{\mu}$ ) is the set of (ground) $\mu$-normal forms of $\mathcal{R}$. We write $s \hookrightarrow^{!} t$ if $t$ is a $\mu$-normal form of $s$, i.e., $s \hookrightarrow^{*} t$ and $t \in \mathrm{NF}_{\mathcal{R}}^{\mu}$; if $s \hookrightarrow^{!} t$ and $s \hookrightarrow^{!} t^{\prime}$ imply $t=t^{\prime}$, then we denote such unique $\mu$-normal form of $s$ as $s \downarrow_{\downarrow_{\mu}}$.

\section{Canonical completeness of context-sensitive rewriting}

Hendrix and Meseguer's presentation concerns order-sorted term rewriting systems modulo a set of equations. We restrict ourselves to TRSs in order to keep the presentation simpler and homogeneous with the previous material. A TRS $\mathcal{R}$ is (ground) $\mu$-confluent if for every (ground) term $s$ such that $s \hookrightarrow^{*} t$ and $s \hookrightarrow^{*} t^{\prime}$, there is a term $u$ such that $t \hookrightarrow^{*} u$ and $t^{\prime} \hookrightarrow^{*} u$ [4]. If $\mathcal{R}$ is (ground) $\mu$-confluent, then every (ground) $\mu$-normal form of a term $t$ is unique; $\mathcal{R}$ is (ground) $\mu$-normalizing if every (ground) term $t$ has a $\mu$-normal form [5].

Definition 1. [3, Definition 6] Let $\mathcal{R}=(\mathcal{F}, R)$ be a TRS and $\mu \in M_{\mathcal{R}}$ be such that $\mathcal{R}$ is ground $\mu$-normalizing and ground $\mu$-confluent. The canonical term algebra for $(\mathcal{R}, \mu)$ is the $\mathcal{F}$-algebra $\operatorname{Can}_{\mathcal{R}}^{\mu}=\left(A, \mathcal{F}_{\text {Can }_{\mathcal{R}}^{\mu}}\right)$ such that $A=\mathrm{GNF}_{\mathcal{R}}^{\mu}$ and for each $f \in \mathcal{F}$, and $t_{1}, \ldots, t_{k} \in A, f_{C_{a n}^{\mu}}^{\mu}\left(t_{1}, \ldots, t_{k}\right)=f\left(t_{1}, \ldots, t_{k}\right) \downarrow_{\mu}$.

When $\operatorname{Can}_{\mathcal{R}}^{\mu}$ is taken as a model to reason about the equivalence of two terms, it turns out that $\operatorname{Can}_{\mathcal{R}}^{\mu}$ is a sound, but not necessarily complete model. The property of TRSs that guarantees the desired completeness (for ground $\mu$-weakly normalizing and ground confluent TRSs, see [3, Theorem 1]) is called $\mu$-canonical completeness and requires that the sets of ground normal forms with respect to unrestricted rewriting and with respect to CSR are equal.

Definition 2. [3, Definition 7] A TRS $\mathcal{R}$ is $\mu$-canonically complete if every ground $\mu$-normal form is a normal form $\left(\mathrm{GNF}_{\mathcal{R}}^{\mu} \subseteq \mathrm{GNF}_{\mathcal{R}}\right)$.

Since normal forms are always $\mu$-normal forms, $\mu$-canonically completeness is equivalent to $\mathrm{GNF}_{\mathcal{R}}^{\mu}=\mathrm{GNF}_{\mathcal{R}}$, i.e., the normal forms and $\mu$-normal forms of $\mathcal{R}$ coincide. An $f$-rule of $\mathcal{R}$ is a rule $\ell \rightarrow r \in \mathcal{R}$ such that $\operatorname{root}(\ell)=f$. Let $\mathcal{R}_{f}$ be the set of $f$-rules of $\mathcal{R}: \mathcal{R}_{f}=\{\ell \rightarrow r \in \mathcal{R} \mid \operatorname{root}(\ell)=f\}$; we say that they define $f$. We say that $\alpha: f\left(\ell_{1}, \ldots, \ell_{k}\right) \rightarrow r \in \mathcal{R}$ has a strict overlap at $i, 1 \leq i \leq k$ if $\ell_{i} \notin \mathcal{X}$ and there is $\alpha^{\prime}: \ell^{\prime} \rightarrow r^{\prime} \in \mathcal{R}$ such that $\mathcal{V}$ ar $(\alpha) \cap \mathcal{V}$ ar $\left(\alpha^{\prime}\right)=\varnothing$ (rename the variables if necessary; $\alpha$ and $\alpha^{\prime}$ can be the same rule) and $\ell_{i}$ and $\ell^{\prime}$ unify.

Theorem 1. Let $\mathcal{R}$ be a TRS and $\mu \in M_{\mathcal{R}}$ be such that $\mathcal{R}$ is $\mu$-canonically complete. Let $f$ be a $k$-ary defined symbol such that $\mu(f) \neq\{1, \ldots, k\}$ and $\mathcal{R}_{f}$ is left-linear. If for some $i \in\{1, \ldots, k\}-\mu(f)$ no $f$-rule has a strict overlap at $i$, then $f$ is completely defined.

Proof. If $f$ were not completely defined, then $\operatorname{root}(t)=f$ for some $t \in \mathrm{GNF}_{\mathcal{R}}$. Let $t=f\left(t_{1}, \ldots, t_{k}\right)$ with $t_{1}, \ldots, t_{k} \in \mathrm{GNF}_{\mathcal{R}}$. Let $i \in\{1, \ldots, k\}-\mu(f)$ be the index of a non- $\mu$-replacing argument of $f$, and $t^{\prime}=f\left(t_{1}, \ldots, t_{i-1}, \sigma(\ell), \ldots, t_{k}\right)$ where $\ell \rightarrow r \in \mathcal{R}$ and $\sigma$ is a substitution given by $\sigma(x)=t$ for all $x \in \mathcal{V}$ ar $(\ell)$. By construction, $t^{\prime} \notin \mathrm{GNF}_{\mathcal{R}}$. By $\mu$-canonical completeness, $t^{\prime} \notin \mathrm{GNF}_{\mathcal{R}}^{\mu}$, i.e., $t^{\prime}$ 
is $\mu$-reducible. Since $t_{1}, \ldots, t_{k} \in \mathrm{GNF}_{\mathcal{R}} \subseteq \mathrm{GNF}_{\mathcal{R}}^{\mu}$ and $i \notin \mu(f)$, a $\mu$-rewriting step at the root position $\Lambda$ is the only possibility. Then, there is $\ell^{\prime} \in L(\mathcal{R})$ and a substitution $\sigma^{\prime}$ such that $t^{\prime}=\sigma^{\prime}\left(\ell^{\prime}\right)$. Since we can assume that $\mathcal{V} a r(\ell) \cap$ $\mathcal{V} a r\left(\ell^{\prime}\right)=\varnothing$ (possibly after some renaming), this means that $\left.\ell^{\prime}\right|_{i}$ and $\ell$ unify. If $\left.\ell^{\prime}\right|_{i} \in \mathcal{X}$, then, by linearity of $\ell^{\prime}$, we would have $\sigma^{\prime \prime}\left(\ell^{\prime}\right)=t$ for some substitution $\sigma^{\prime \prime}$, thus contradicting that $t \in \mathrm{GNF}_{\mathcal{R}}$. If $\left.\ell^{\prime}\right|_{i} \notin \mathcal{X}$, then $\ell^{\prime}$ has a strict overlap with $\ell$ at position $i$, leading again to a contradiction.

Corollary 1. Let $\mathcal{R}$ be a constructor TRS and $\mu \in M_{\mathcal{R}}$ be such that $\mathcal{R}$ is $\mu$ canonically complete. Let $f$ be a $k$-ary defined symbol. If $\mu(f) \neq\{1, \ldots, k\}$ and $\mathcal{R}_{f}$ is left-linear, then $f$ is completely defined.

Example 2. Symbol if in Example 1 is not completely defined (e.g., if $(0,0,0) \in$ $\left.\mathrm{GNF}_{\mathcal{R}}\right)$. By Corollary 1, the TRS is not $\mu$-canonically complete.

A left-linear TRS is called orthogonal if there are no rules $\ell \rightarrow r$ and $\ell^{\prime} \rightarrow r^{\prime}$ with a nonvariable position $p \in \mathcal{P}_{o S_{\mathcal{F}}}(\ell)$ such that $\left.\ell\right|_{p}$ and $\ell^{\prime}$ unify with $m g u$ $\sigma$. The case $\ell \rightarrow r=\ell^{\prime} \rightarrow r^{\prime}$ and $p=\Lambda$ is excluded. For orthogonal TRSs $\mathcal{R}$, requiring $\mu \in C M_{\mathcal{R}}$ is necessary to achieve $\mu$-canonical completeness.

Theorem 2. Let $\mathcal{R}$ be an orthogonal TRS such that $\mathrm{GNF}_{\mathcal{R}} \neq \varnothing$, and $\mu \in M_{\mathcal{R}}$. If $\mathcal{R}$ is $\mu$-canonically complete, then $\mu \in C M_{\mathcal{R}}$.

Proof. If $\mu \notin C M_{\mathcal{R}}$, then $\mu_{\mathcal{R}}^{\text {can }} \nsubseteq \mu$ and there is $\ell \in L(\mathcal{R})$ and $p \in \mathcal{P}_{\text {os }}(\ell)$ such that $p \notin \mathcal{P o s}^{\mu}(\ell)$. Note that $p>\Lambda$. Since $\mathrm{GNF}_{\mathcal{R}} \neq \varnothing$, there is a constant $a \in \mathcal{C}$. Let $t=\sigma\left(\ell[\ell]_{p}\right) \in \mathcal{T}(\mathcal{F})$ where $\sigma$ is a substitution given by $\sigma(x)=a$ for all $x \in \operatorname{Var}(\ell)$. Clearly, $t$ is reducible at position $p$ (i.e., $t \notin \mathrm{GNF}_{\mathcal{R}}$ ). We prove that $t \in \mathrm{GNF}_{\mathcal{R}}^{\mu}$ by contradiction. Assume that $\sigma^{\prime}\left(\ell^{\prime}\right)=\left.t\right|_{q}$ for some $q \in \mathcal{P}_{o s}{ }^{\mu}(t)$, $\ell^{\prime} \rightarrow r^{\prime} \in \mathcal{R}$ and substitution $\sigma^{\prime}$. We have two cases for $q: q \| p$ and $q<p$ (the case $q \geq p$ is not possible because $p \notin \mathcal{P}_{o s}{ }^{\mu}(t)$ implies $\left.q \notin \mathcal{P}_{\text {os }}{ }^{\mu}(t)\right)$.

1. If $q \| p$, then $q \neq \Lambda$ and $\left.t\right|_{q}=\left.\sigma(\ell)\right|_{q}=\sigma^{\prime}\left(\ell^{\prime}\right)$. We have $q \in \mathcal{P}_{\operatorname{Pos}}(\ell)$; otherwise, there would be a variable $x \in \mathcal{P}$ os $(\ell)$ such that $\left.t\right|_{q}$ is a subterm of $\sigma(x)$, i.e., $\left.t\right|_{q}=a$ (by definition of $\sigma$ ), which is a normal form. Thus, $\left.t\right|_{q}=\sigma\left(\left.\ell\right|_{q}\right)=\sigma^{\prime}\left(\ell^{\prime}\right)$ and, since we can assume $\mathcal{V} \operatorname{ar}(\ell) \cap \mathcal{V} \operatorname{ar}\left(\ell^{\prime}\right)=\varnothing$, we conclude that $\ell^{\prime}$ and $\left.\ell\right|_{q}$ unify and $\mathcal{R}$ has a strict overlap, a contradiction.

2. If $q<p$, we can write $p=q \cdot p^{\prime}$ for some position $p^{\prime}$. Note that, since $p \notin$ $\mathcal{P}_{o s}^{\mu}(t)$ and $q \in \mathcal{P}_{\text {os }}^{\mu}(t)$, we have $p^{\prime} \neq \Lambda$. Furthermore, $p^{\prime} \notin \mathcal{P}_{\text {os }}{ }^{\mu}\left(\left.t\right|_{q}\right)$. Therefore, $\sigma^{\prime}\left(\ell^{\prime}\right)=\sigma\left(\left.\ell\right|_{q}\right)[\sigma(\ell)]_{p^{\prime}}$. We consider two cases for $p^{\prime}$ :

(a) If $p^{\prime} \in \mathcal{P}$ os $\left(\ell^{\prime}\right)$ (but note that $p^{\prime} \notin \mathcal{P}_{o s}{ }^{\mu}\left(\ell^{\prime}\right)$ ), then $\left.\ell^{\prime}\right|_{p^{\prime}}$ and $\ell$ unify and $\mathcal{R}$ has a strict overlap, leading to a contradiction.

(b) If $p^{\prime} \notin \mathcal{P} \operatorname{os}\left(\ell^{\prime}\right)$, then there is $q^{\prime} \in \mathcal{P}_{\text {os }}\left(\ell^{\prime}\right)$ such that $q^{\prime}<p^{\prime}$. By leftlinearity of $\mathcal{R}$, we can define a substitution $\sigma^{\prime \prime}$ such that $\sigma^{\prime \prime}\left(\ell^{\prime}\right)=$ $\sigma\left(\left.\ell\right|_{q}\right)$. Thus, $\left.\ell\right|_{q}$ and $\ell^{\prime}$ unify and $\mathcal{R}$ is overlapping, leading to a contradiction unless we have $q=\Lambda$ and $\ell=\ell^{\prime}$. In this case, $\sigma^{\prime}(\ell)=$ $\sigma(\ell)[\sigma(\ell)]_{p}=\sigma\left(\ell[\ell]_{p}\right)$, i.e., $\ell$ has a strict overlap, also a contradiction.

Thus, $t \in \mathrm{GNF}_{\mathcal{R}}^{\mu}-\mathrm{GNF}_{\mathcal{R}}$ contradicting $\mu$-canonical completeness of $\mathcal{R}$. 
Definition 3. [4] Given a set of symbols $\mathcal{B}, \mu_{\mathcal{B}}(f)=\{1, \ldots$, ar $(f)\}$ for all $f \in \mathcal{B}$, and $\mu_{\mathcal{B}}(f)=\varnothing$ if $f \notin \mathcal{B}$. We let $\mu_{\mathcal{R}}^{\mathcal{B}}=\mu_{\mathcal{R}}^{\text {can }} \sqcup \mu_{\mathcal{B}}$.

Note that, for all sets of symbols $\mathcal{B}, \mu_{\mathcal{R}}^{\mathcal{B}} \in C M_{\mathcal{R}}$. The following result shows that $\mu$-canonical completeness actually requires that all arguments of all constructor symbols of the TRS be $\mu$-replacing.

Theorem 3. Let $\mathcal{R}=(\mathcal{F}, R)=(\mathcal{C} \uplus \mathcal{D}, R)$, be a TRS such that $\mathrm{GNF}_{\mathcal{R}} \neq \varnothing$, and $\mu \in M_{\mathcal{R}}$. If $\mathcal{R}$ is $\mu$-canonically complete, then $\mu_{\mathcal{C}} \sqsubseteq \mu$.

Proof. By contradiction. Let $c \in \mathcal{C}$ be such that $\mu(c) \neq\{1, \ldots, \operatorname{ar}(c)\}$ and $i \in\{1, \ldots, \operatorname{ar}(c)\}-\mu(c)$. For $t=c(a, \ldots, \underbrace{\sigma(\ell)}_{i}, \ldots, a)$ where $\ell \in L(\mathcal{R}), a \in \mathcal{C}$

is a constant and $\sigma(x)=a$ for all $x \in \operatorname{V}$ ar $(\ell)$, we have $t \in \mathrm{GNF}_{\mathcal{R}}^{\mu}-\mathrm{GNF}_{\mathcal{R}}$, contradicting $\mu$-canonical completeness of $\mathcal{R}$.

Example 3. The following TRS $\mathcal{R}$ can be used to compute approximations to $\frac{\pi}{2}$ as $\frac{\pi}{2}=\lim _{n \rightarrow \infty} \frac{2}{1} \frac{2}{3} \frac{4}{3} \frac{4}{5} \cdots \frac{2 n}{2 n-1} \frac{2 n}{2 n+1}$ (Wallis' product):

$$
\begin{aligned}
& \text { evenNs } \rightarrow 0: \operatorname{incr}(\operatorname{oddNs}) \\
& \text { oddNs } \rightarrow \text { incr(evenNs) } \\
& \operatorname{incr}(x: x s) \rightarrow \mathrm{s}(x): \operatorname{incr}(x s) \\
& \operatorname{take}(0, x s) \rightarrow \text { nil } \\
& \operatorname{take}(\mathrm{s}(n), x: x s) \quad \rightarrow \quad x: F \text { take }(n, x s) \\
& \operatorname{zip}(\text { nil, } x s) \quad \rightarrow \quad \text { nil } \\
& \operatorname{zip}(x s, \mathrm{nil}) \quad \rightarrow \quad \text { nil } \\
& \operatorname{zip}(x: x s, y: y s) \rightarrow \frac{x}{y}: \operatorname{zip}(x s, y s) \\
& \operatorname{tail}(x: x s) \rightarrow x s \\
& \operatorname{rep} 2(\text { nil }) \rightarrow \text { nil } \\
& \operatorname{rep} 2(x: x s) \rightarrow x: x: \operatorname{rep} 2(x s) \\
& 0+x \rightarrow x \\
& s(x)+y \quad \rightarrow \quad \mathrm{s}(x+y) \\
& 0 \times y \rightarrow 0 \\
& s(x) \times y \quad \rightarrow \quad y+(x \times y) \\
& \frac{x}{y} \times \div \frac{z}{t} \rightarrow \frac{x \times z}{y \times t} \\
& \Pi(\text { nil }) \rightarrow \frac{\mathrm{s}(0)}{\mathrm{s}(0)} \\
& \Pi\left(p:_{F} p s\right) \quad \rightarrow \quad p \times \div \Pi(p s) \\
& \operatorname{halfPi}(n) \rightarrow \Pi(\operatorname{take}(n, \operatorname{zip}(\operatorname{rep} 2(\operatorname{tail}(\operatorname{evenNs})), \operatorname{tail}(\operatorname{rep} 2(\operatorname{oddNs})))))
\end{aligned}
$$

A call halfPi $\left(\mathrm{s}^{n}(0)\right)$ for some $n>0$ returns the desired approximation. Although $\mathcal{R}$ is nonterminating (due to the first two rules), with $\mu$ given by $\mu(:)=\varnothing$ and $\mu(f)=\{1, \ldots, \operatorname{ar}(f)\}$ for all $f \in \mathcal{F}-\{:\}, \mathcal{R}$ is $\mu$-terminating and this makes useful computations possible (see Example 6 below). However, since $\mu(:)=\varnothing$, we have $\mu_{\mathcal{C}} \nsubseteq \mu$ and Theorem 3 proves that $\mathcal{R}$ is not $\mu$-canonically complete.

Corollary 2. Let $\mathcal{R}$ be an orthogonal TRS such that $\mathrm{GNF}_{\mathcal{R}} \neq \varnothing$, and $\mu \in M_{\mathcal{R}}$. If $\mathcal{R}$ is $\mu$-canonically complete, then $\mu_{\mathcal{R}}^{\mathcal{C}} \sqsubseteq \mu$.

Theorem 4. $[4$, Theorem 11] Let $\mathcal{R}=(\mathcal{F}, R)=(\mathcal{C} \uplus \mathcal{D}, R)$ be a left-linear TRS, $\mathcal{B} \subseteq \mathcal{C}$, and $\mu \in M_{\mathcal{R}}$ be such that $\mu_{\mathcal{R}}^{\mathcal{B}} \sqsubseteq \mu$. For all $t \in \mathcal{T}(\mathcal{F}, \mathcal{X})$ and $\delta \in \mathcal{T}(\mathcal{B}, \mathcal{X})$, we have $t \rightarrow^{*} \delta$ iff $t \hookrightarrow^{*} \delta$.

Example 4. By Theorem 4, the evaluation of fact $\left(\mathrm{s}^{n}(0)\right)$ by using CSR with $\mathcal{R}$ and $\mu$ in Example 1 yields $\mathrm{s}^{n !}(0)$ because $\mu_{\mathcal{R}}^{\mathcal{C}} \sqsubseteq \mu$. Since $\mathcal{R}$ is $\mu$-terminating, all values $\mathrm{s}^{n !}(0)$ associated to initial expressions fact $\left(\mathrm{s}^{n}(0)\right)$ can be obtained in finite time. And terms like fact $(\mathrm{p}(0))$ do not start any infinite computation. 
Comparing Corollary 2 and Theorem 4, we see that, for orthogonal TRSs, $\mu$ canonical completeness requires replacement maps $\mu$ that are less restrictive than the ones that are necessary to obtain values of initial expressions. For instance, $\mathcal{R}$ in Example 1 is not $\mu$-canonically complete. However, Example 4 shows the use of $\mu$ to obtain the intended normal forms for the application.

\section{1. $\mu$-normal forms as normal forms}

The set of $\mu$-ground terms is the set of terms having no variables in replacing positions, i.e., $G T^{\mu}=\left\{t \in \mathcal{T}(\mathcal{F}, \mathcal{X}) \mid M R C^{\mu}(t) \in \mathcal{T}(\mathcal{F} \cup\{\square\})\right\}[6$, Definition 9]. Nakamura, Ogata and Futatsugi give the following definition.

Definition 4. [6, Definition 12] Let $\mathcal{R}$ be a TRS. Then, $\mu \in M_{\mathcal{R}}$ is $\mu$-ground correct if $\mathrm{NF}_{\mathcal{R}}^{\mu} \cap G T^{\mu} \subseteq \mathrm{NF}_{\mathcal{R}}$.

However, $\mu$-ground correctness implies $\mu$-canonical completeness of $\mathcal{R}$.

Proposition 1. Let $\mathcal{R}$ be a TRS and $\mu \in M_{\mathcal{R}}$. If $\mu$ is $\mu$-ground correct, then $\mathcal{R}$ is $\mu$-canonically complete.

Proof. If $\mathrm{NF}_{\mathcal{R}}^{\mu} \cap G T^{\mu} \subseteq \mathrm{NF}_{\mathcal{R}}$, then $\mathrm{NF}_{\mathcal{R}}^{\mu} \cap G T^{\mu} \cap \mathcal{T}(\mathcal{F})=\mathrm{NF}_{\mathcal{R}}^{\mu} \cap \mathcal{T}(\mathcal{F})=$ $\mathrm{GNF}_{\mathcal{R}}^{\mu} \subseteq \mathrm{NF}_{\mathcal{R}} \cap \mathcal{T}(\mathcal{F})=\mathrm{GNF}_{\mathcal{R}}$, i.e., $\mathcal{R}$ is $\mu$-canonically complete.

Therefore, all previous necessary conditions for $\mu$-canonical completeness are valid for $\mu$-ground correctness as well. In particular, we have.

Corollary 3. Let $\mathcal{R}$ be an orthogonal TRS such that $\mathrm{GNF}_{\mathcal{R}} \neq \varnothing$, and $\mu \in M_{\mathcal{R}}$. If $\mu$ is $\mu$-ground correct, then $\mu_{\mathcal{R}}^{\mathcal{C}} \sqsubseteq \mu$.

Without orthogonality, this result does not hold.

Example 5. Consider the following TRS [6, Section 5.1]:

$$
\begin{array}{rlrl}
0+x & \rightarrow x & x+0 & \rightarrow x \\
\mathrm{~s}(x)+y & \rightarrow \mathrm{s}(x+y) & y+\mathrm{s}(x) & \rightarrow \mathrm{s}(x+y)
\end{array}
$$

Clearly, $\mu_{\mathcal{R}}^{\text {can }}(+)=\{1,2\}$. However, with $\mu(\mathrm{s})=\{1\}$ and $\mu(+)=\{1\}$ (or $\mu(+)=\{2\}$ ), $\mu$ is $\mu$-ground correct (see [6, Theorem 14]). Note that $\mu \notin C M_{\mathcal{R}}$.

Since $\mu$-ground correctness implies $\mu$-canonical completeness (Proposition 1), Example 5 also shows that having $\mu \in C M_{\mathcal{R}}$ is not necessary for $\mu$-canonical completeness of non-orthogonal (but left-linear TRSs).

\subsection{Semantic completeness of CSR}

Semantic completeness of CSR guarantees that the algebraic semantics $\mathrm{Can}_{\mathcal{R}}^{\mu}$ is isomorphic to the standard, mathematical semantics of $\mathcal{R}$ [3, Theorem 4].

Definition 5. [3, Definition 8] A TRS $\mathcal{R}$ is $\mu$-semantically complete if for all $s, t \in \mathcal{T}(\mathcal{F}), s \downarrow_{\mu}=t_{\downarrow_{\mu}}$ if and only if $s=\mathcal{R}_{\mathcal{R}} t$.

Since Hendrix and Meseguer prove that ground weakly normalizing and $\mu$ semantically complete TRSs $\mathcal{R}$ are $\mu$-canonically complete [3, Theorem 6], our results apply to ground weakly normalizing $\mu$-semantically complete TRSs $\mathcal{R}$. 


\section{Sufficient completeness of CSR}

Theorem 3 entails that the intended use of replacement restrictions to forbid reductions on distinguished arguments of constructor symbols thus enforcing a terminating behavior (as in Example 3) makes $\mu$-canonical completeness unattainable in many practical cases. Sufficient completeness of CSR gives semantic support to this practical aspect of CSR: "to obtain a terminating method to execute a nonterminating rewrite system" [3, Section 4.3].

Definition 6. [3, Definition 9] $A$ TRS $\mathcal{R}=(\mathcal{F}, R)=(\mathcal{C} \uplus \mathcal{D}, R)$ is $\mu$-sufficiently complete relative to $\mathcal{B} \subseteq \mathcal{C}$ if for all $t \in \mathrm{GNF}_{\mathcal{R}}^{\mu}, M R C^{\mu}(t) \in \mathcal{T}(\mathcal{B} \cup\{\square\})$. If $\mathcal{B}=\mathcal{C}$, we just say that $\mathcal{R}$ is $\mu$-sufficiently complete.

Theorem 5. Let $\mathcal{R}=(\mathcal{F}, R)=(\mathcal{C} \uplus \mathcal{D}, R)$ be a TRS and $\mu \in M_{\mathcal{R}}$. If $\mathcal{R}$ is $\mu$-sufficiently complete with respect to $\mathcal{B} \subseteq \mathcal{C}$, then $\mathcal{R}$ is completely defined.

Proof. If there is $t \in \mathrm{GNF}_{\mathcal{R}}$ with $\operatorname{root}(t)=f \in \mathcal{D}$, then $t \in \mathrm{GNF}_{\mathcal{R}}^{\mu}$ but $M R C^{\mu}(t)=f\left(t_{1}, \ldots, t_{k}\right)$ for some terms $t_{1}, \ldots, t_{k} \in \mathcal{T}(\mathcal{F} \cup\{\square\})$. Since $f \notin \mathcal{B}$, this contradicts sufficient completeness of $\mathcal{R}$.

Example 6. Note that $\mathcal{R}$ in Example 3 is not completely defined (e.g., $\Pi(0) \in$ $\mathrm{GNF}_{\mathcal{R}}$ ); hence it is not $\mu$-sufficiently complete (Theorem 5). If the evaluation of halfPi $\left(\mathrm{s}^{n}(0)\right)$ yields $\frac{\mathrm{S}^{p}(0)}{\mathbf{S}^{q}(0)}$ (approximating $\frac{\pi}{2}$ as $\frac{p}{q}$ ), Theorem 4 ensures that we can obtain it with CSR: the subset of constructors involved in such terms is $\mathcal{B}=\{0, \mathrm{~s}$, 号 $\} \subseteq\left\{0\right.$, nil, $\left.\mathrm{s},:,:_{F}, \frac{\square}{\square}\right\}=\mathcal{C}$, and $\mu_{\mathcal{R}}^{\mathcal{B}} \sqsubseteq \mu$ holds. Since $\mathcal{R}$ is (provably) $\mu$-terminating, we can use CSR to obtain such output expressions in finite time.

Orthogonal $\mu$-sufficient complete TRSs $\mathcal{R}$ must use $\mu \in C M_{\mathcal{R}}$.

Theorem 6. Let $\mathcal{R}$ be an orthogonal TRS such that $\mathrm{GNF}_{\mathcal{R}} \neq \varnothing$, and $\mu \in M_{\mathcal{R}}$. If $\mathcal{R}$ is $\mu$-sufficiently complete, then $\mu \in C M_{\mathcal{R}}$.

Proof. If $\mu \notin C M_{\mathcal{R}}$, then $\mu_{\mathcal{R}}^{\text {can }} \nsubseteq \mu$ and there is $\ell \rightarrow r \in \mathcal{R}$ and $p \in \mathcal{P}_{\text {os }}(\ell)$ such that $p \notin \mathcal{P} s^{\mu}(\ell)$. Note that $p>\Lambda$. Let $t=\sigma\left(\ell[\ell]_{p}\right) \in \mathcal{T}(\mathcal{F})$ where $\sigma$ is a substitution given by $\sigma(x)=a$ for all $x \in \mathcal{V}$ ar $(\ell)$ (where, as in the proof of Theorem 3, $a \in \mathcal{C}$ is a constant constructor symbol). Since $\operatorname{root}\left(M R C^{\mu}(t)\right)=$ $\operatorname{root}(\ell) \notin \mathcal{C}$, by $\mu$-sufficient completeness, $t \notin \mathrm{GNF}_{\mathcal{R}}^{\mu}$. By orthogonality of $\mathcal{R}$, any $\mu$-reduction on $t$ must be at the root position. Otherwise, as in the proof of Theorem 2, there would be a strict overlap between $\ell \rightarrow r$ and another rule in $\mathcal{R}$. Thus, there is a rule $\ell^{\prime} \rightarrow r^{\prime} \in \mathcal{R}$ and a substitution $\sigma^{\prime}$ such that $t=\sigma^{\prime}\left(\ell^{\prime}\right)$. Note that $\ell^{\prime} \rightarrow r^{\prime}$ is different from $\ell \rightarrow r$. Otherwise, we would have $\sigma\left(\ell[\ell]_{p}\right)=\sigma^{\prime}(\ell)$, i.e., $\left.\sigma^{\prime}(\ell)\right|_{p}=\sigma(\ell)$, witnessing the existence of an internal overlap on $\ell \rightarrow r$. But if $\ell^{\prime} \rightarrow r^{\prime}$ is different from $\ell \rightarrow r$ and $\sigma^{\prime}\left(\ell^{\prime}\right)=\sigma\left(\ell[\ell]_{p}\right)$, then we consider two cases: If $p \in \mathcal{P}_{\operatorname{Pos}_{\mathcal{F}}}\left(\ell^{\prime}\right)$, then $\left.\sigma^{\prime}\left(\ell^{\prime}\right)\right|_{p}=\sigma(\ell)$, i.e., $\ell \rightarrow r$ overlaps $\ell^{\prime} \rightarrow r^{\prime}$ at position $p$. If $p \notin \mathcal{P o s}_{\mathcal{F}}\left(\ell^{\prime}\right)$, then there is $q<p$ such that $\left.\ell^{\prime}\right|_{q}=x \in \mathcal{X}$. Then, $\left.\sigma^{\prime}\left(\ell^{\prime}\right)\right|_{q}=\sigma^{\prime}(x)=\left.\sigma\left(\ell[\ell]_{p}\right)\right|_{q}$ and, by linearity of $\ell^{\prime}$, we have $\sigma^{\prime \prime}\left(\ell^{\prime}\right)=\sigma(\ell)$ if we let $\sigma^{\prime \prime}(x)=\sigma\left(\left.\ell\right|_{q}\right)$ and $\sigma^{\prime \prime}(y)=\sigma(y)$ for all $y \in \mathcal{V} a r\left(\ell^{\prime}\right)-\{x\}$. In both cases an overlap between $\ell^{\prime} \rightarrow r^{\prime}$ and $\ell \rightarrow r$ contradicts orthogonality of $\mathcal{R}$. 


\section{Conclusions and future work}

We have shown that the semantic notions introduced in $[3,6]$ agree with our previous analysis of completeness of CSR with canonical replacement maps: both $\mu$-canonical and $\mu$-sufficient completeness imply $\mu \in C M_{\mathcal{R}}$ for orthogonal TRSs $\mathcal{R}$ (Theorems 2 and 6 ). For $\mu$-canonical completeness, all arguments of all constructor symbols of the TRS must be $\mu$-replacing (Theorem 3). The same applies to the $\mu$-ground correct replacement maps in [6]. And being completely defined is also necessary (at different levels) for TRSs $\mathcal{R}$ that enjoy the considered semantic properties (Theorems 1 and 5). In practice, though, requiring $\mu$ to fit the full-powered semantic notions lead to more restrictive computational settings than required in $[4,5]$ (Examples 4 and 6).

The analysis in [3] concerns order-sorted TRSs. The sort discipline can be seen as another restriction mechanism on the structure of terms which is useful to discard some situations which hinder completeness. For instance, the sort discipline is helpful to make TRSs completely defined. This could lead to further refinements in the definition of the canonical replacement map for sorted TRSs [6, Sections 3.4 and 3.5]. On the other hand, our analysis of computations of head-normal forms and infinite values and normal forms in $[4,5]$ is missing (but envisaged) in [3]. This is also connected with some algebraic semantics for infinite rewriting and the notion of productivity of rewriting specifications, which has been recently investigated by a number of authors. These interesting issues deserve further investigation which we intend to address in the future.

\section{References}

[1] M. Clavel, F. Durán, S. Eker, P. Lincoln, N. Martí-Oliet, J. Meseguer, and C. Talcott. All About Maude - A High-Performance Logical Framework, LNCS volume 4350. Springer-Verlag, 2007.

[2] K. Futatsugi and A. Nakagawa. An Overview of CAFE Specification Environment In Proc. of 1st International Conference on Formal Engineering Methods, 1997.

[3] J. Hendrix and J. Meseguer. On the Completeness of Context-Sensitive OrderSorted Specifications. In Proc. of RTA'07, LNCS 4533:229-245, 2007.

[4] S. Lucas. Context-sensitive computations in functional and functional logic programs. Journal of Functional and Logic Programming, 1998(1):1-61, 1998.

[5] S. Lucas. Context-Sensitive Rewriting Strategies. Information and Computation, 178(1):293-343, 2002.

[6] M. Nakamura, K. Ogata, and K. Futatsugi. Reducibility of operation symbols in term rewriting systems and its application to behavioral specifications. Journal of Symbolic Computation, 45:551-573, 2010. 